\title{
Experimentelle Analyse von Last- und Treibketten zur Optimierung der Lebensdauer
}

\section{Experimental Analysis of Load and Drive Chains to Optimize the Product Life}

\author{
Christian Häfner \\ Markus Schröppel \\ Institut für Fördertechnik und Logistik \\ Abteilung Maschinenentwicklung und Materialflussautomatisierung \\ Universität Stuttgart
}

$\mathbf{Z}$ usammen mit einem namhaften Kettenhersteller hat das Institut für Fördertechnik und Logistik (IFT) im Rahmen eines AIF-geförderten Forschungsprojektes einen Kettenverschleißprüfstand für Last- und Treibketten entwickelt und gebaut, um neuentwickelte Ketten in kurzen Testphasen unter normierter Umgebung auf ihre Lebensdauer bzw. Verschleißfestigkeit zu prüfen. Durch steigende Rohstoffkosten und den immer größer werdenden Konkurrenzdruck im Bereich der Ketten entsteht ein hoher Bedarf an der Entwicklung neuer leistungsfähigerer Produkte. Die Neuheit dieser Projektidee liegt in dem erstmaligen Einsatz alternativer Werkstoffe und Konstruktionen in Kombination mit neuen verschleißarmen Beschichtungsverfahren für Kettenbauteile um Schmierstoffe langfristig einzulagern und bedarfsgerecht abzugeben.

[Schlüsselwörter: Kettenverschleißprüfstand, Lastketten, Treibketten, Lebensdauer, Verschleißfestigkeit, Beschichtungsverfahren]

$\mathbf{T}$ ogether with a well-known chain manufacturer, the Institute for Material Handling and Logistics (IFT) developed and built a chain wear test rig for load and drive chains in an AIF funded research project to check newly developed chains in short test phases under a standardized environment for their life and wear resistance. Due to rising raw material costs and the ever increasing competitive pressure in the area of chains there is a great need for the development of new more efficient products. The novelty of this project idea is the first use of alternative materials and construction combined with new wear-resistant coating processes for long-chain components to store lubricants in the long run and to release them demand-actuated.

[Keywords: chain wear test, load chains, drive chains, life resistance, wear resistance, coating process]

\section{EINLEITUNG}

In der Intralogistik werden in Stetig- und Unstetigförderern Ketten als Zug- oder Treibmittel eingesetzt. Aufgrund mangelnder Forschung der letzten 40 Jahre im Bereich der Optimierung von Flyer- und Rollenketten ist eine Aufarbeitung des Themas notwendig. Ketten als „altes“ Basiselement sollen mit modernen Mitteln weiterentwickelt und verbessert werden.

Speziell werden Lastketten (Flyerketten im Gabelstapler als Hubkette), die nur als Zugorgan zur Umlenkung von Kräften dienen und Treibketten (Rollenketten in Kettentrieben), die Drehmoment über Zahnräder übertragen, getestet (siehe Abbildung 1).

Unterscheidung der Ketten nach dem Einsatzfall*

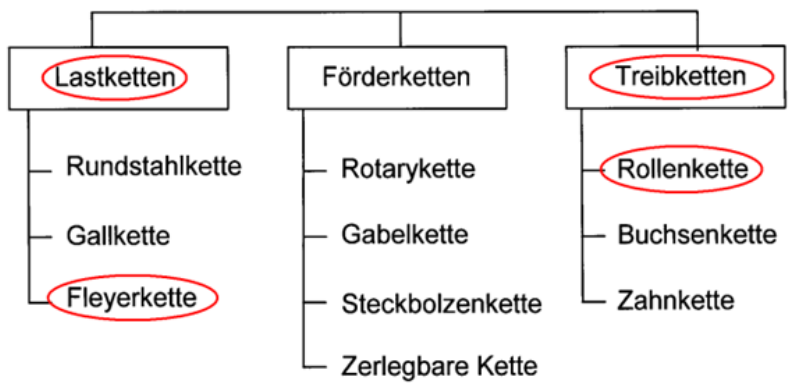

*Ketten werden z. T. für unterschiedliche Einsatzfälle genutzt

Abbildung 1. Unterscheidung Ketten

Die Lebensdauer von Last- und Treibketten wird durch die Abnutzung in den Gelenkstellen und durch Korrosion erschöpft. Ein Bruch erfolgt plötzlich. Um die genauen Einflussgrößen unter den speziellen Betriebsbedingungen zu bestimmen, muss der Einfluss folgender Medien und Belastungen im Bereich der fördertechnischen Maschinen und Anlagen auf die Ketten mit einem Prüfstand untersucht werden: 
- Umgebungsbedingungen (Temperatur, Säuren)

- Aussetz-/Reversierbetrieb

- extreme Lastkollektive

- ungleichförmige Schwingungen und Stöße

- Kettengeschwindigkeiten

- Vorspannkräfte

- Schmierstoffverhalten

Aufgrund der stark zunehmenden Leistungsanforderungen an Ketten und Kettentriebe ist zur weiteren Leitungssteigerung bzw. Lebensdauererhöhung ein noch leistungsfähigerer Werkstoff als die heute schon üblichen hochlegierten Stähle notwendig. Hier wird eine ingenieurwissenschaftliche Vorgehensweise entwickelt, die aus den Elementen:

- Systematische Aufnahme des Ist-Zustandes und Analyse,

- Methodische Konstruktion,

- Innovationsidee,

- Experimentelle Untersuchungen sowie

- Rechnergestützte numerische Analyse

zu einem nachvollziehbaren reproduzierbaren Optimierungsverfahren für Ketten führt und dessen Anwendung im Rahmen des Projektes zu den geforderten, neuartigen Produkten führt.

Mit Hilfe eines ganzheitlichen Ansatzes für Hubsysteme mit Lastketten (Flyerkette über Umlenkrollen) in Flurförderzeugen und Antriebssystemen mit Treibketten (Rollenkette über Zahnräder) in Stetig-/ Unstetigförderern, sollen innovative Lösungsansätze gefunden werden, um bestehende Zielkonflikte z. B. zwischen Korrosionsschutz, Verschleißfestigkeit, Wartungsfreiheit, Geräuschminderung, Material-/ Energieeinsparungen und der Verringerung der Umweltbelastung zu überwinden. Wirtschaftlich und technisch realisierbares Verbesserungspotential wird bei allen Kettenkomponenten erwartet (z. B. durch Variation/ Optimierung der Werkstoffe, der Beschichtungen, der Schmierstoffe, den Kettengeometrien, den Fertigungs- und Herstellungsverfahren usw.).

Die erkannten Grenzen und Schwachstellen der heutigen Ketten werden im folgenden im Detail beschrieben und die wirtschaftliche Bedeutung für die deutsche Herstellerbranche dargestellt. Dies macht die grundsätzliche Notwendigkeit einer Optimierung der Ketten offensichtlich. Mit dieser Produktoptimierung würde die Möglichkeit geschaffen durch neue Kettenwerkstoffe/ -beschichtungen die Lebensdauer und z. B. die Anzahl der Hubspiele von Gabelstaplern so zu steigern, dass ein Al- leinstellungsmerkmal für diese Einsatzbedingungen erzeugt wird.

Das Fehlen von Grundlagenuntersuchungen muss aufgearbeitet werden und wird dadurch belegt, dass die Anzahl der wissenschaftlichen Untersuchungen sehr klein ist und die wenigen Dissertationen zu diesem Thema mehrere Jahrzehnte alt sind. Moderne Verfahren wie FEM und MKS (Mehrkörpersimulation) und neue Erkenntnisse im Bereich Werkstoffkennwerte und Analysen, z. B. Kinematik und Verschleiß, wurden bisher noch nicht berücksichtigt. Im Projekt sollen diese Verfahren angewandt werden und die Erkenntnisse in die Lebensdaueranalyse mit eingearbeitet werden.

\section{Projektziele}

Die Neuheit der Projektidee liegt in dem Einsatz alternativer Werkstoffe und Beschichtungen mit langfristiger Schmierstoffeinlagerung für Kettenbauteile zur Erhöhung der Lebensdauer. Bisher erschien dieser Einsatz neuer Werkstoffe zu teuer. Durch steigende Rohstoffkosten und den immer größer werdenden Konkurrenzdruck ist Handlungsbedarf zur Verbesserung der Produkte notwendig. Durch den Einsatz alternativer Materialien/ Beschichtungen/ Schmierstoffe sollen die Kettennachschmierintervalle verlängert werden, wodurch weniger Kettenwechsel notwendig sind. Das langfristige Ziel ist der Einsatz von wartungsfreien und verschleißarmen Ketten. Wartungsarme und wartungsfreie Ketten reduzieren über die Produktlebenszeit die Wartungskosten erheblich, so dass sich für den Kunden ein Mehrwert (Minderaufwand) einstellt, der sich gegenüber den höheren Bezugskosten rechnet.

Ziel des Forschungsprojekts ist es, die Lebensdauer von Ketten als wichtiges Basiselement technischer Logistiksysteme (z. B. beim Einsatz in Flurförderzeugen und Stetig- sowie Unstetigförderern) unter Beibehaltung der Produktionskosten gravierend zu verlängern, indem durch Korrosion auftretender Verschleiß drastisch reduziert wird und die Verwendung nicht-ressourcenschonender Materialien vermindert wird. Reproduzierbare Auslegungs-, Berechnungs- und Testverfahren für verschiedene Einsatzzwecke sollen zusätzlich generiert werden.

Ebenfalls im Fokus des Projektes steht die Erhöhung der Materialeffizienz durch die Entwicklung oder Kombination neuer Materialien. Durch die Entwicklung und Kombination neuer Materialien soll vor allem eine Verringerung des Gelenkverschleißes und der Materialschädigung durch Korrosion und damit die Verlängerung der Standzeiten von Bauteilen und Anlagen (Lebensdauererhöhung) ermöglicht werden.

Die Entwicklung neuer Materialien sieht einerseits den Einsatz korrosionsbeständiger Werkstoffe im Bereich der Treibketten und der niederbeanspruchten Lastketten 
vor. Andererseits ist für hochbeanspruchte Lastketten aus jetziger Sicht der Grundwerkstoff Stahl noch unumgänglich. Hier gilt es, neue Beschichtungen zu entwickeln, die sowohl die Ketten vor Korrosion schützen, als auch Schmierstoffe für die gesamte Lebensdauer der Ketten in die Gelenkverbindungen einlagern. Durch diese Kombination aus Korrosionsschutz und Schmiermitteleinlagerungsvermögen soll eine wartungsfreie (!) Kette für den hochbeanspruchten Belastungssektor entwickelt werden, die die gleichen Anforderungen wie Edelstahlketten bei halbierten Materialmengen und -kosten erfüllen.

Die angestrebte Erhöhung der Standzeit und die Steigerung der Ressourceneffizienz von Last- und Treibketten durch den Einsatz alternativer verschleißarmer und korrosionsbeständiger Materialien soll durch die Erreichung folgender Teilziele sichergestellt werden:

- Verringerung der Materialschädigung durch Korrosion und damit Verlängerung der Standzeiten durch Erhöhung der Verschleißfestigkeit von Flyer- und Rollenketten

- Erhöhung der Sicherheit von Bauteilen und Anlagen durch verbesserten Korrosionsschutz und Erhöhung der ertragbaren Lasten (Bruchkraft, Dauerfestigkeit)

- $\quad$ Erhöhung der ertragbaren Lasten (Bruchkraft, Dauerfestigkeit)

- Verlängerung der Wartungsintervalle (mit dem langfristigen Ziel der Wartungsfreiheit)

- Verringerung von Umweltbelastungen durch nachhaltige Korrosionsschutzschichten und Verhinderung des Austritts von Schmierstoffen

- $\quad$ Geräuschminderung

- $\quad$ Durch Anpassung der Korrosionsschutzkonzepte und Verminderung der Gelenkreibung folgt die Effizienzsteigerung bei der Energieübertragung durch Kettentriebe

Im Rahmen des Projektes sollen allgemeingültige normierte Berechnungs- und Dimensionierungsverfahren zur einfacheren und wirtschaftlicheren Auslegung von Ketten und Kettentrieben geschaffen werden. Eine präzisere Kettendimensionierung soll in verschiedenen Anwendungsfällen (z. B. Lastketten im FFZ) den Einsatz von kleineren Kettengrößen gestatten, wodurch sich erhebliche Material- und Kosteneinsparungspotentiale ergeben.

Durch die Verbesserung bzw. Einführung von praxisgerechten Beiwerten bei der Dimensionierung und Lebensdauerberechnung von Treib- und Lastketten sollen sich die Verschleiß- und Belastungskriterien bei der Kettenauslegung genauer bestimmen lassen, um somit die hohen Sicherheitsbeiwerte zu verringern. Durch verbesserte Auswahlverfahren für Ketten können der Konstruk- tionszeitraum und die entstehenden Kosten verringert werden. Dies erhöht die Konkurrenzfähigkeit deutscher Unternehmen. Kettentriebe können dann ohne umfangreiche, anwendungsabhängige Vorversuche ausgelegt werden.

Aus jahrzehntelanger Forschung im Bereich Seile und aktuell auch im Bereich Räder und Rollen ist erkennbar, dass in der Optimierung der Basiselemente ein großes Potential steckt. Die wirtschaftliche Notwendigkeit das Basiselement „Kette“ zu optimieren, führt systemtechnisch zu einer Effizienzsteigerung der gesamten intralogistischen Maschinen und Anlagen.

Das Ergebnis des Projektes und folgender Forschungsprojekte soll einen wesentlichen Schritt auf dem Weg zur Weiterentwicklung zu einer verschleißfreien Kette darstellen. Weiterhin soll durch die Verringerung der Kettenabmaße neben einer möglichen Geräuschreduzierung (auch durch die Senkung des Polygoneffektes) eine Einsparung von Bauraum und damit die Reduzierung der bewegten Massen realisiert werden, was besonders bei Rollenketten eine Steigerung der Energieeffizienz zur Folge hätte.

Um nachzuweisen, ob alternative Werkstoffe und Beschichtungen für Kettenbauteile wirtschaftlich eingesetzt werden können, wurde im Rahmen des Forschungsprojektes am IFT ein Kettenverschleißprüfstand entwickelt und gebaut. Dadurch lassen sich die neu entwickelten Ketten im Vergleich zur Praxis in enorm kürzerer Zeit auf ihre Lebensdauer testen.

\section{KetTEnAUSWAHL}

Bei der Auswahl einer Kette für den jeweiligen Einsatzbereich müssen mehrere Faktoren beachtet werden (siehe Abbildung 2). Die wichtigste Größe zur Dimensionierung einer Kette ist die Kenntnis über die Gesamtbelastung. Diese setzt sich je nach Einsatzfall und Kettentyp aus mehreren Faktoren zusammen, der Kettenzugkraft, der Kettengeschwindigkeit, der Stoßbelastung, dem Polygoneffekt, den Schwingungen und den Umgebungseinflüssen (Vgl. Abbildung 3).

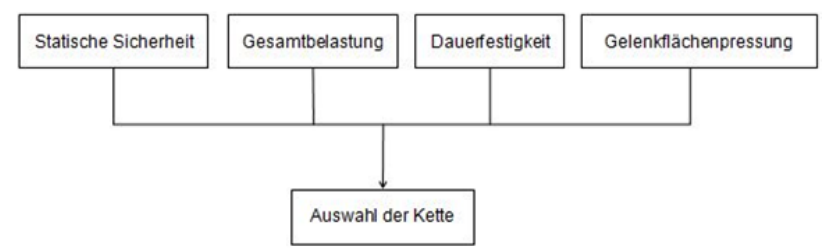

Abbildung 2. Einflussfaktoren Kettenauswahl

Unter Umgebungseinflüssen fallen beispielsweise Säuren, Reinigungsmittel, Staub, Wärme, Schmierung und sonstige Umwelteinflüsse, denen die Kette im Betrieb ausgesetzt ist. Da Umgebungseinflüsse kaum rechnerisch zu bestimmen sind, lässt sich die Gesamtbelastung nur bis 
zu einem gewissen Grad exakt bestimmen. Der Polygoneffekt verursacht eine ungleichförmige Kettenbewegung.

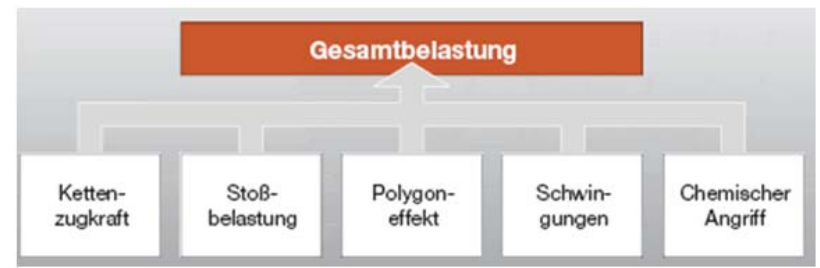

Abbildung 3. Gesamtbelastung einer Flyerkette [Rex03]

Stoßbelastungen, die von außen in das System induziert werden, müssen in der Regel abgeschätzt oder überschlägig berechnet werden. Diese können beispielweise beim Überfahren von Unebenheiten oder einer Kante mit einem Gabelstapler entstehen. Schwingungen als schwellende zusätzliche Kettenbelastung können durch Fremderreger erzeugt werden, beispielsweise bei Antriebsmotoren von Werkzeugmaschinen oder bei Gabelstaplern bei bestimmten Fahrbahnzuständen.

Bei der Auswahl der Kette ist neben der Verschleißfestigkeit auch die Dauerfestigkeit in Abhängigkeit der verschiedenen Betriebsbedingungen sicherzustellen (siehe Abbildung 4). Anhand der ertragbaren Bruchkraft kann keine Aussage über die Betriebssicherheit bzw. die Dauerfestigkeit der Kette getroffen werden.



Abbildung 4. Einflussfaktoren zur Lebensdauerbestimmung [Rex03]

Wird die Kette bis nah an die Elastizitätsgrenze belastet, so besteht die Gefahr dass Ermüdungsbrüche bereits nach wenigen Lastwechseln auftreten. Diese Ermüdungsbrüche treten meist ohne vorhergehende plastische Verformung auf. Erfolgt die Kettenbelastung allerdings im Zeitfestigkeitsbereich, bei sachgemäßer Handhabung, so wird die Lebensdauer ausschließlich durch die Ver- schleißfestigkeit bestimmt. Abbildung 5 zeigt die verschiedenen Festigkeitsbereiche nach Wöhler.

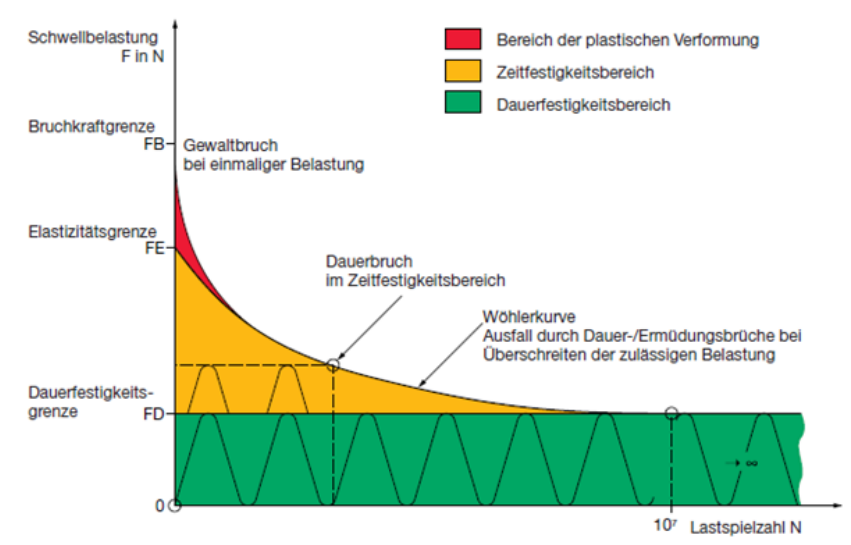

Abbildung 5. Dauerfestigkeitsschaubild nach Wöhler [Rex03]

Beim Einsatz von Flyerketten in Gabelstaplern bis 10 t Tragkraft müssen die Ketten beispielsweise einen statischen Sicherheitsbeiwert von 5 aufweisen. Das bedeutet, die Bruchkraft der Kette muss um das 5-fache größer sein als die max. Kettenbelastung. Da zwischen der maximalen Bruchkraft und der Dauerfestigkeit ein Zusammenhang besteht, ist die Dimensionierung allein über den Weg der statischen Sicherheit unzureichend, da dies zu einer Unterdimensionierung der Kette im Bereich der Dauerfestigkeit führen kann und dies eine unwirtschaftliche Lösung zur Folge hat.

Ein weiterer Punkt, der berücksichtigt werden muss, ist die Flächenpressung in den Kettengelenken. Eine durch große Belastungen hervorgerufene hohe Gelenkflächenpressung kann zu einem hohem Verschleiß durch Kettenlängung und in Verbindung mit großen Laschenkombinationen zu Kaltverschweißungen in den Kettengelenken führen.

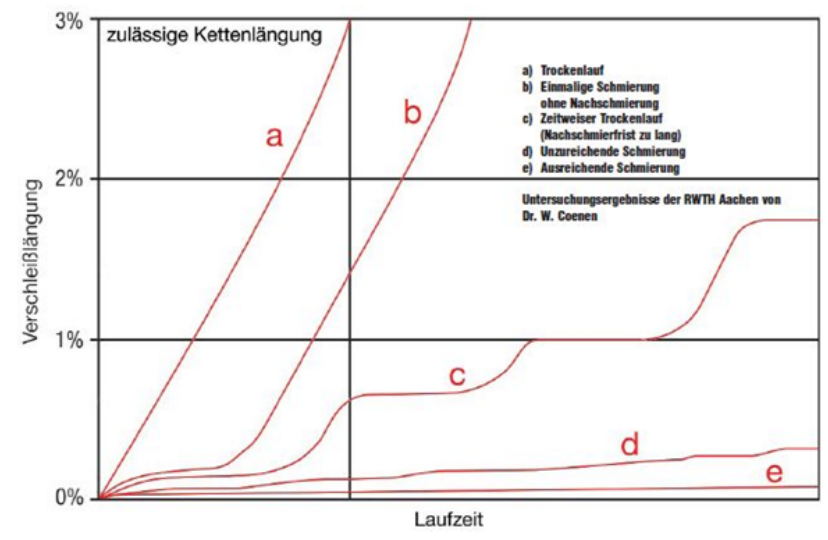

Abbildung 6. Einfluss der Kettenschmierung auf die Lebensdauer von Rollenketten [Coe84] 


\section{SCHÄDIGUNGSARTEN}

Die Hauptursache für Verschleiß ist Reibung und Korrosion. Reibung entsteht durch eine Relativbewegung zwischen zwei Körpern zueinander. Es wird zwischen Roll-, Haft,- und Gleitreibung unterschieden. Je nach Art der vorherrschenden Reibung treten verschiedene Verschleißerscheinungen, wie Adhäsions- und Abrasionserscheinungen oder Zerrüttungsverschleiß auf.

Bei Flyerketten existiert eine Reibpaarung zwischen Innenlaschen und Kettenbolzen. Durch das Einpressen der Bolzen in die Außenlaschen findet dort kein Verschleiß statt. Abbildung 7 zeigt das Verhalten von Bolzen und Innenlasche beim Überlauf über eine Umlenkrolle.



Abbildung 7. Reibpaarung Bolzen/Lasche

Beim Auflaufen auf die Umlenkrolle rollt der Bolzen so lange an der Innenfläche der Lasche ab, bis ein Gleichgewicht zwischen dem Reibmoment und dem Knickmoment, das durch die an der Umlenkrolle wirkende Umfangskraft hervorgerufen wird, herrscht. Dann geht die Rollbewegung in eine Gleitbewegung über. Beim Ablaufen von der Umlenkrolle wirkt das gleiche Prinzip. Dieser so genannte Gelenkverschleiß bewirkt, dass der Bolzen nicht mehr mittig in der Lasche läuft. Dadurch erfahren die Außenlaschen eine höhere Belastung, die sich in einer Vergrößerung der Teilung des Außengliedes niederschlägt (siehe Abbildung 8). Durch das Auf- und Ablaufen der Ketten auf Umlenkrollen, sowie durch den Polygoneffekt hervorgerufene Schwingungen herrscht im Kettengelenk eine oszillierende Beanspruchung.

\subsection{MATERIALSCHÄDIGUNG DURCH KORROSION}

Europaweit werden jährlich neue Flyerketten mit einem Gesamtvolumen von $18.000 \mathrm{t}$ verkauft, wobei ein Viertel der Flyerketten als korrosionsbeständige Ketten ausgeführt sind. Im laufenden Projekt wird dieser Anteil deutlich ausgebaut, wobei das Verhältnis zwischen zu erzielender Lebensdauer und dem Preis einen entscheidenden Anteil am Markterfolg haben wird. Gleiches gilt für den Bereich der Rollenketten, der mit insgesamt $40.000 \mathrm{t}$ pro Jahr allein in Europa und 4.000 t / 8.000 t bei korrosions- beständigen sowie verzinkten Ketten ein noch erheblich größeres Volumen hat als der Markt für Flyerketten.
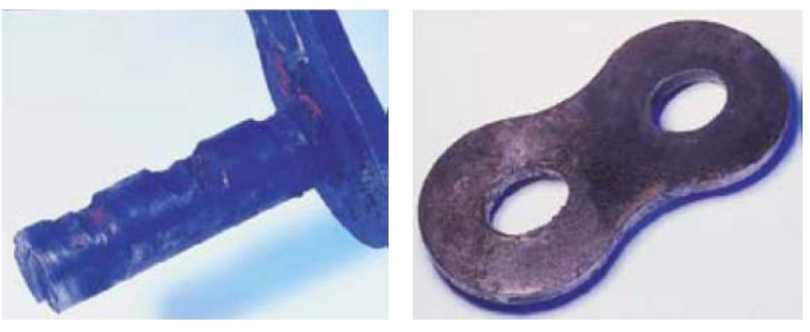

Abbildung 8. Verschleiß Kettenbolzen und in Laschenbohrung

In korrosionsgefährdeten Umgebungen werden von den Anwendern von Flurförderzeugen und Stetigförderern je nach Korrosionsschutzklasse Ketten verlangt, die korrosionsbeständig sind und die gleichen hohen Standzeiten erreichen sollen, wie nicht korrosionsbeständige Standardketten. Dies ist technisch aus heutiger Sicht nur bedingt möglich, denn die Kettenhersteller sehen für diese Einsatzfälle Edelstahlketten vor. Der größte Nachteil von Edelstahlketten liegt in der geringeren Lebensdauer aufgrund der verminderten Zugfestigkeit des Werkstoffs und des daraus resultierenden schnelleren und höheren Verschleißes. Die Kettenhersteller neigen aus diesem Grund bewusst dazu, die Edelstahlketten überzudimensionieren, um eine hohe Kettenlebensdauer zu erreichen. Die so entstehenden Kosten für korrosionsbeständige Edelstahlketten liegen aus den genannten Gründen in etwa doppelt so hoch wie bei Standardketten aus Vergütungsstahl.

Könnten die Standardketten aus Vergütungsstahl korrosionsbeständig hergestellt werden, würde der Materialmehraufwand im Vergleich zu Edelstahlketten reduziert werden oder könnte sogar vollständig entfallen. Der Energieaufwand bei der Herstellung von Standardstahl im Vergleich zu Edelstahl ist ebenfalls deutlich geringer. Somit würden zum einen Materialressourcen geschont und zum anderen die erforderliche Energie im Herstellungsprozess deutlich reduziert.

In zahlreichen Industriezweigen werden in Stetigund Unstetigförderer Rollenketten aus Edelstahl eingesetzt. Die Umgebungsbedingungen sind oftmals sehr feucht und häufig auch säurebelastet. Ein klassisches Beispiel sind Förderer in der Lebensmittelindustrie, die über Ketten Waren transportieren. In hygienisch unkritischen Bereichen könnten hier auch Standardrollenketten aus Vergütungsstahl eingesetzt werden, sofern diese die Anforderungen an die Korrosionsbeständigkeit erfüllen.

In Flurförderzeugen (z. B. Gabelstaplern) die in korrosionsgefährdeten Umgebungen zum Einsatz kommen, werden in den Hubmasten der Stapler Lastketten (Flyerketten) aus Edelstahl eingesetzt. Dieser Anwendungsfall tritt in zahlreichen Industriezweigen auf, die auch von feuchten und säurehaltigen (korrosiongefährdeten) Umgebungsbedingungen betroffen sind, wie z. B. in Hafenum- 
schlagsgebieten und in der Fisch- und Lebensmittelindustrie. Ein großer Teil der Flurförderzeuganwender im Outdoorbereich setzt hier dennoch (unbewusst) nicht korrosionsbeständige Ketten ein. Diese Ketten unterliegen höheren Schmierintervallen, um zumindest einen Oberflächenschutz gegen Korrosion aufzubringen. Meist werden die notwendigen Nachschmierintervalle in der Praxis nicht eingehalten und die Ketten müssen deshalb aufgrund von auftretendem Korrosionsverschleiß vorzeitig abgelegt werden.

Die im Rahmen dieses Projektes zu optimierenden technischen Basiselemente „Lastkette von In-/OutdoorFlurförderzeugen“ und „Treibkette von Stetig-/ Unstetigförderern“ unterliegen außerordentlich extremen Verschleiß- und Ausfallbedingungen. Obwohl für die bei Flurförderzeugen zum Einsatz kommenden Lastketten eine Lebensdauer zwischen 2 und 3 Jahren angestrebt wird, ist heute festzustellen, dass durch die sich bei den Anwendern in den letzten Jahren immer weiter verschärfenden Belastungen teilweise nur noch Standzeiten von weniger als einem Jahr (in Extremfällen z. B. bei den Lastketten von Gabelstaplern im Hafenumschlagsbetrieb und in der Fischindustrie Lebensdauern von nur 700 - 900 Betriebsstunden im Mehrschichtbetrieb) erreicht werden.

Als Ursachen für diese außerordentlich kurzen Standzeiten sind zu nennen:

- Die Lebensdauer der Ketten wird erschöpft durch die Abnutzung in den Gelenkstellen. Diese Abnutzung hängt maßgeblich vom Verschleiß und von dem Einfluss der Korrosion ab.

- Materialschädigung durch Rost aufgrund ungünstiger Umgebungsbedingungen

- Salz, Hitze und säurehaltige Umgebungen sind die maßgebliche Ursache für hohe Korrosion

- Erhöhter Abrieb durch Schlupf aus der Reibpaarung Kette/ Umlenkrolle

- Thermische Überlastungen der Ketten durch mangelnde Schmiermittelversorgung infolge hoher Verschmutzung der Kettenglieder

- Verschmutzung verhindert Eintritt von Schmiermittel in die Kette und fördert Verschleiß und Korrosion

- Hohe Umgebungstemperaturen verursachen Schmiermittelaustritt aus Kette

- Die geringere Härte und Zugfestigkeit von Edelstahl im Vergleich zu Standardstahl führt trotz Materialmehraufwand zu vorzeitiger Kettenablegereife aufgrund von Verschleiß
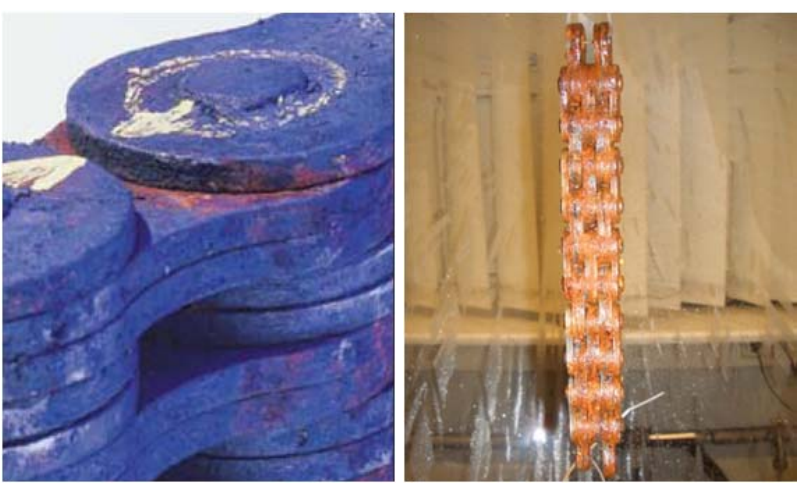

Abbildung 9. Gelenk- und Oberflächenrost bei Flyerketten

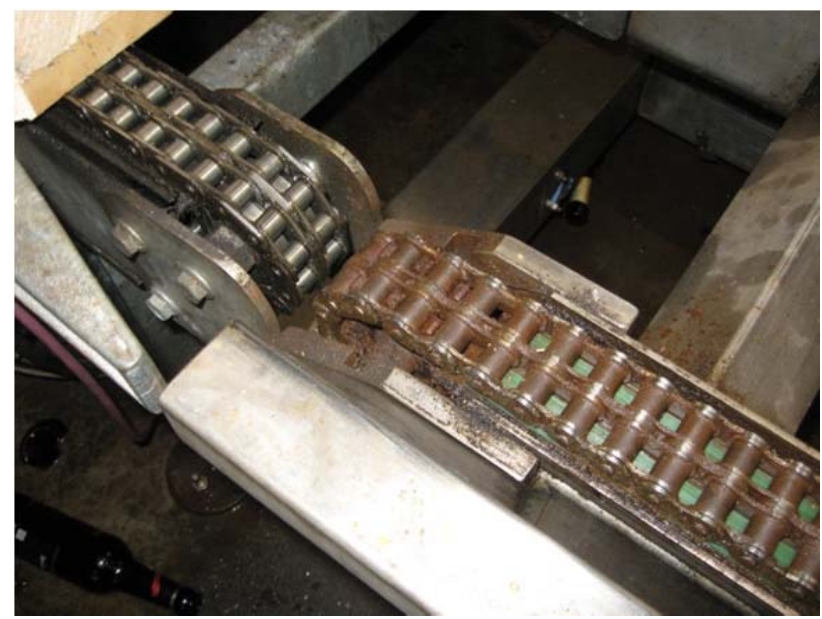

Abbildung 10. Vergleich links neue Rollenkette/ rechts korrodierte Rollenkette



Abbildung 11. Gelenk- und Oberflächenrost bei Rollenketten 


\section{KETTENBETRIEBSGRÖßEN/ \\ KETTENVERSCHLEIßPRÜFSTAND}

Um die Kettenbetriebsgrößen zu bestimmen, müssen die Ketten mittels einer Dauerprüfbelastung untersucht werden. Die Untersuchung der Dauerbelastung läuft nach folgendem Konzept ab. Die Kette wird über zwei angetriebene Umlenkrollen geführt und entsprechend der im Betrieb zu erwartenden Belastung verspannt.

Folgende Parameter werden durch Sensoren bzw. komplexe Messtechnik aufgezeichnet und ausgewertet:

- Umlaufgeschwindigkeit

- Kraftverlauf der Vorspannung

- Permanente Messung der Kettenlänge/ Vorspannkraft

- $\quad$ Temperatur der Kette/ Umlenkrolle/ Lager/ Antriebsmotoren

- Drehzahlen

- Drehmomente

Abbildung 12 zeigt den schematischen Aufbau eines solchen Prüfverfahrens. Im Dauerbetrieb werden so die Betriebsstunden bis zur Ablegereife bzw. zum Versagen der Kette ermittelt.

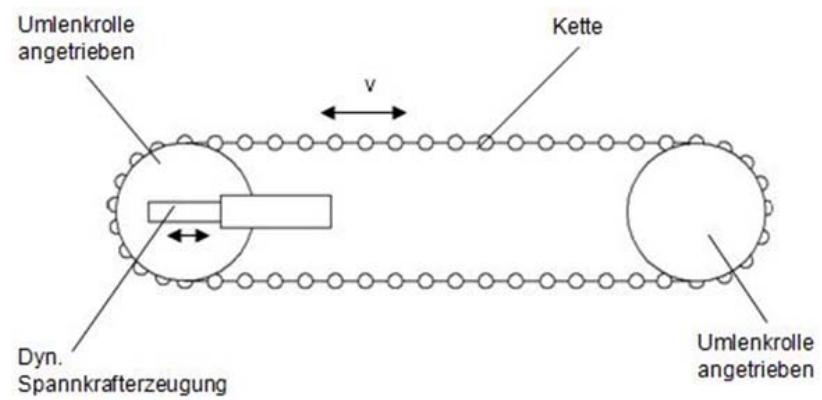

Abbildung 12. Schema Dauerbelastungsprüfung

Durch das Aufzeichnen der Messparameter können Lebensdauerkurven verschiedener Kettenarten und -werkstoffe in Bezug auf die Beiwerte erstellt werden. Mittels einer Dauerprüfbelastung werden die neu entwickelten Ketten unter realitätsnahen Umgebungsbedingungen untersucht, indem sie über zwei angetriebene Umlenkrollen geführt werden und entsprechend der im Betrieb zu erwartenden Belastungen verspannt werden.

Durch den Umlauf kürzerer Ketten erfahren beim Prüflauf die einzelnen Kettengelenke mehr Gelenkbiegungen, wodurch sich die Prüfdauer erheblich verkürzen lässt. Dadurch lassen sich die neu entwickelten Ketten im Vergleich zur Praxis in enorm kürzerer Zeit auf ihre Lebensdauer testen. Ein durchschnittlicher Prüflauf dauert somit maximal 10 Tage, bis die Ablegereife (ab 2-3\% Kettenlängung) der Kette erreicht ist. Die Kette wird pneumatisch vorgespannt um Stöße aus dem Polygoneffekt durch das kompressible Medium Luft zu dämpfen.

Programmierbare Lastkollektive können während der Prüfläufe unterschiedliche Vorspannkräfte/ Geschwindigkeiten von statischer bis zur dynamischen Beanspruchung auf die Kette generieren. Der Reversierbetrieb ist dadurch ebenfalls realisierbar und kann bspw. die Auf/ Ab-Hubbewegung eines Gabelstaplerhubmastes simulieren, indem die Kette vorwärts und rückwärts bewegt wird.

Mit Hilfe des Prüfstandes (siehe Abbildung 13 und 14) können messtechnisch folgende Daten während der Testläufe erfasst und visualisiert werden:

- $\quad$ die Kettennabenvorspannkraft $(0-130 \mathrm{kN})$ über eine Zugkraftmessdose

- die Kettenverschleißlängung über ein Magnetbandwegmesssystem (bei 2-3\% Längung ist die Ablegereife erreicht)

- die Kettengeschwindigkeit $(0-2 \mathrm{~m} / \mathrm{s})$ über die Drehzahl der Umlenkrollen/Zahnräder

- $\quad$ die Reibung (bzw. Schlupf) zwischen Umlenkrolle und Kette über Drehmomentmesswellen

- die Kettentemperatur (Wärmebildkamera)

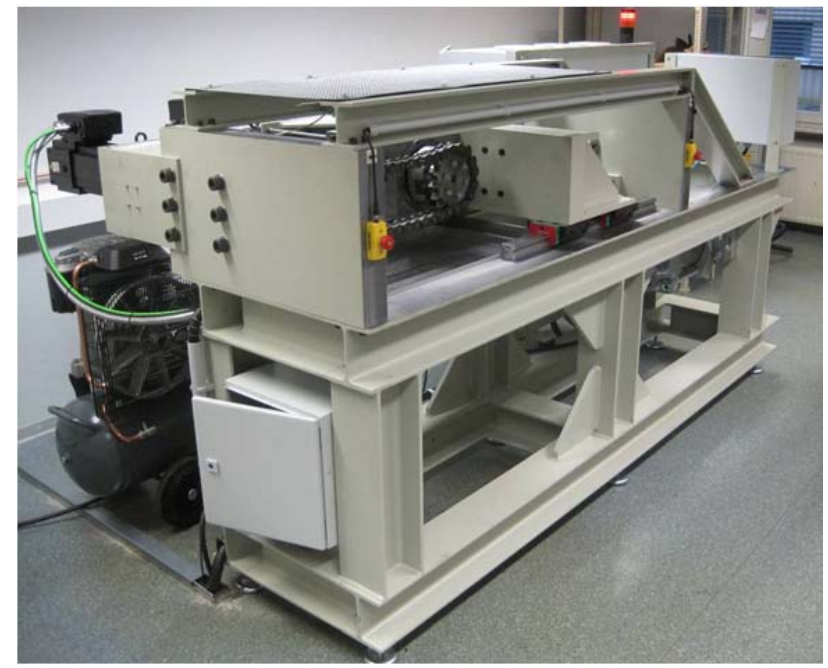

Abbildung 13. Kettenverschleißprüfstand IFT 


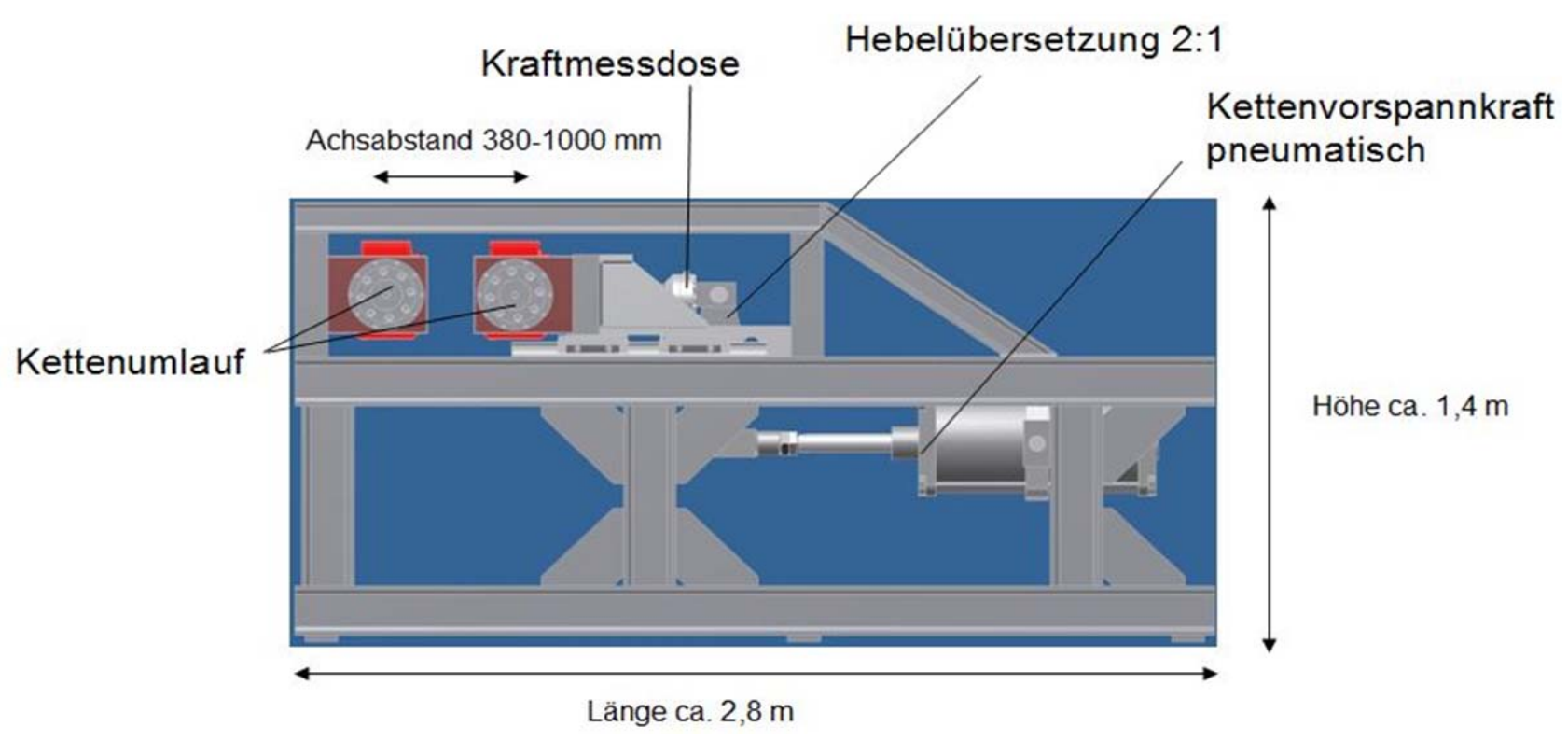

Abbildung 14. Kettenverschleißprüfstand IFT (CAD)

\section{ZuSAmmenfassung UND AUSBLICK}

Aufgrund der ständig wachsenden Anforderungen an die Verfügbarkeit der in den Produktionsprozess eingebundenen Stetig- und Unstetigförderer auf bis zu 100\% und der auch weiterhin notwendigen Steigerung der Umschlagsleistung müssen die Ausfallzeiten und -kosten der Fahrzeuge und Förderer infolge Kettenversagen und Verschleiß durch Korrosion minimiert werden.

Durch die zu entwickelnde innovative, neue Kettentechnologie werden verschiedene Optimierungsgesichtspunkte gleichzeitig untersucht, um eine zukunftsträchtige optimale Lösung zu finden:

- Alternative Konstruktionen von Ketten

- Alternative Entwicklung von Werkstoffen, Beschichtungen und Schmierstoffen

- Alternative Herstellungsverfahren

- Einlagerung von Schmierstoffen in die Werkstoffe und Beschichtungen für eine Lebensdauerschmierung

- Verzicht auf den Einsatz von Edelstahlketten

Mit dem Kettenverschleißprüfstand wurde ein Werkzeug geschaffen, um Ketten in kurzen Testphasen unter realitätsnahen Umgebungsbedingungen zu testen. Basierend auf ersten Versuchen konnten bereits durch einfache konstruktive Änderungen von Flyerketten im Vergleich zu herkömmlichen typgleichen Flyerketten die Standzeit auf dem Prüfstand um über 20\% erhöht werden. Dies zeigt das Optimierungspotential und bestätigt den Bedarf an der Weiterentwicklung leistungsfähigerer Ketten.

\section{LITERATUR}

[Coe84] Coenen, Wilfried: Einfluß der Schmierung auf das Verschleißverhalten von Rollenketten. Dissertation der RWTH Aachen, 1984

[Rex03] Rexnord Kette Betzdorf: Die Technik der Flyerkette. 2003/2004

[Rex06] Rexnord Kette Betzdorf: Qualitätsketten. 2006 